\title{
Eficacia y seguridad del aborto medicamentoso auto-administrado versus administrado por profesionales de la salud
}

\author{
Efficacy and safety of self-administered versus provider-administered medical abortion
}

\section{Comentado de:}

Gambir K, et al. Cochrane Database Syst Rev. 2020 Mar 9;3(3):CD013181. PMID: $32150279^{1}$

\section{Objetivos}

Comparar la eficacia, seguridad y aceptabilidad del aborto medicamentoso autoadministrado versus el administrado por un profesional de la salud en cualquier ámbito (clínica, hospital u hogar), en mujeres entre 15 a 49 años de edad de estratos sociales bajos, medios o altos, que buscaban un aborto inducido y hubieran dado su consentimiento informado.

\section{Fuente de datos}

Se realizaron búsquedas hasta julio de 2019 en las siguientes bases de datos electrónicas con el fin de identificar estudios relevantes: Registro Cochrane Central de Ensayos Controlados (Ovid EBM Reviews), MEDLINE en curso y otras citas no indexadas, Embase, CINAHL Plus, POPLINE, LILACS, ClinicalTrials.gov, Plataforma Internacional de Registro de Ensayos Clínicos de la Organización Mundial de la Salud (ICTRP), y Google Scholar (incluyendo ensayos recientes aún no indexados en las principales bases de datos). También se contactaron expertos en el campo para obtener datos adicionales y se realizaron búsquedas en los siguientes sitios web: Marie Stopes International, Ipas, Gynuity Health Projects, Population Council y el Consorcio Internacional para el Aborto Médico. No se aplicaron restricciones por idioma.

\section{Selección de estudios}

Los criterios de inclusión de los estudios incluyeron: comparar aborto médico autoadministrado vs. administrado por profesionales de la salud, tener una evaluación prospectiva de los resultados, e incluir mujeres con edades entre 15 a 49 años que buscaran un aborto inducido.

\section{Extracción de datos}

Los datos considerados de interés fueron extraídos por dos revisores independientes mediante una herramienta adaptada a los criterios de inclusión: edad gestacional media, edad media de las participantes, gravidez y paridad previa, estado civil, nivel de instrucción, pérdidas durante el seguimiento, medicamentos utilizados (mifepristona, misoprostol), dosis y cantidad de las mismas, vías de administración, modalidad de administración (institución de salud, hogar), administración por profesionales en instituciones de salud, cantidad de mujeres reclutadas en cada grupo, comparación y cantidad de abortos completos, proporción de mujeres con abortos exitosos, complicaciones, efectos secundarios, aceptabilidad, entre otros.
Por otro lado, los revisores contactaron a los investigadores de los estudios primarios para obtener datos adicionales sobre los métodos y/o los resultados según fuera necesario.

\section{Medidas de desenlace}

Se establecieron dos tipos de desenlaces por medio de los cuales se valoró la eficacia, la seguridad y la aceptabilidad de la intervención realizada.

Para evaluar la eficacia se definieron un desenlace primario, el aborto exitoso (entendido como la evacuación uterina completa sin necesidad de intervención quirúrgica) y un desenlace secundario, el embarazo en curso (definido como la progresión clínica o por un método de imagen del embarazo en dos semanas de seguimiento).

Los desenlaces secundarios fueron la ocurrencia de cualquier complicación que requiera intervención quirúrgica y la aceptabilidad (definida como el nivel de satisfacción de la mujer con el método medicamentoso, la probabilidad de elegirlo nuevamente o de recomendarlo a una amiga).

El aborto medicamentoso se definió como aquel que implica la utlización de mifepristona oral seguida de misoprostol 24 a 48 horas después.

\section{Resultados principales}

Se incluyeron 18 estudios que cumplían los criterios de elegibilidad; 16 eran de tipo observacional (estudios de cohorte) y dos, ensayos clínicos controlados aleatorizados (ECCA).

Los resultados principales se detallan en la Tabla 1 y la Tabla 2. En cuanto a la aceptabilidad de ambos métodos, evidencia proveniente de ensayos no aleatorizados documentó que $91,2 \%$ de las participantes del grupo aborto autoadministrado y $90,5 \%$ del grupo supervisado tuvieron satisfacción con el método recibido, $83,4 \%$ de las participantes del grupo aborto autoadministrado lo volverían a elegir, frente a $53 \%$ del grupo supervisado, mientras que $87,2 \%$ de las participantes en el grupo de aborto autoadministrado lo recomendarían a otras personas, frente a $52 \%$ de quienes tuvieron supervisión de un profesional de la salud durante el procedimiento.

\section{Conclusiones}

Esta revisión sistemática muestra que la autoadministración de la segunda etapa del esquema medicamentoso mifepristonamisoprostol presenta la misma eficacia que la administración por profesionales de la salud. Sin embargo, no aporta suficiente evidencia para determinar su seguridad. Por otro lado, ha permitido mostrar que la autoadministración fue altamente aceptable.

Conflicto de interés de los autores: No referidos. 
Tabla 1. Aborto médico autoadministrado en comparación con el aborto médico administrado por el proveedor para mujeres en edad reproductiva que buscan aborto inducido, síntesis de los ensayos clínicos aleatorizados. Notas: IC: intervalo de confianza

\begin{tabular}{|l|c|c|c|c|}
\hline Desenlaces & $\begin{array}{c}\text { Aborto autoadministrado, \% } \\
(\mathrm{n} / \mathrm{N})\end{array}$ & $\begin{array}{c}\text { Aborto administrado por } \\
\text { profesionales de la salud, } \% \\
(\mathrm{n} / \mathrm{N})\end{array}$ & $\begin{array}{c}\text { Riesgo Relativo } \\
(\text { IC } 95 \%)\end{array}$ & $\begin{array}{c}\text { Certeza de } \\
\text { Evidencia }\end{array}$ \\
\hline Aborto Exitoso & $96,06(439 / 457)$ & $96,32(446 / 462)$ & $0,99(0,97$ a 1,01) & Moderada \\
\hline Embarazo en curso & $1,37(5 / 365)$ & $0,81(3 / 370)$ & $1,69(0,41$ a 7,02$)$ & Baja \\
\hline
\end{tabular}

Tabla 2. Aborto médico autoadministrado en comparación con el aborto médico administrado por el proveedor para mujeres en edad reproductiva que buscan aborto inducido, síntesis de los ensayos clínicos no aleatorizados. Notas: IC: intervalo de confianza

\begin{tabular}{|l|c|c|c|c|}
\hline Desenlaces & $\begin{array}{c}\text { Aborto } \\
\text { autoadministrado, \% (n/N) }\end{array}$ & $\begin{array}{c}\text { Aborto administrado por } \\
\text { profesionales de la salud, \% (n/N) }\end{array}$ & Riesgo Relativo (IC 95\%) & $\begin{array}{c}\text { Certeza de } \\
\text { Evidencia }\end{array}$ \\
\hline Aborto Exitoso & $93,76(7559 / 8062)$ & $94(1939 / 2062)$ & $0,99(0,97$ a 1,01) & Muy baja \\
\hline Embarazo en curso & $1,7(86 / 5174)$ & $0,8(12 / 1517)$ & $1,28(0,65$ a 2,49) & Muy baja \\
\hline $\begin{array}{l}\text { Complicaciones que requie- } \\
\text { ran tratamiento quirúrgico }\end{array}$ & $4,4(82 / 1880)$ & $2,6(15 / 572)$ & $2,14(0,80$ a 5,71$)$ & Muy Baja \\
\hline
\end{tabular}

\section{Comentario}

Se entiende por aborto inseguro a aquellos procedimientos efectuados por personas que carecen de las habilidades necesarias o realizados en entornos peligrosos para finalizar un embarazo no deseado ${ }^{2}$. Este representa un importante problema de salud pública evitable, ya que hoy en día se cuenta con numerosos métodos abortivos efectivos y seguros, entre ellos los de tipo medicamentoso.

En la revisión resumida, se valoró la evidencia existente sobre el uso de misoprostol y mifepristona con el propósito de determinar si las personas gestantes obtienen la misma eficacia y seguridad al autoadministrarse esta combinación farmacológica para la interrupción voluntaria del embarazo con respecto a hacerlo bajo la supervisión de un profesional de salud. Si así fuera, se podría mejorar el acceso a los métodos abortivos seguros, sobre todo en entornos con escasez de profesionales sanitarios y en los que el aborto representa un estigma social y la violencia institucional ocurre con frecuencia.

Es importante señalar que la presencia de sesgos ha afectado la certeza de la evidencia sintetizada, disminuyendo la confianza en las estimaciones obtenidas. De esta forma, la evidencia para el desenlace principal, el aborto exitoso, fue de certeza moderada, debido a que los resultados provinieron de ECCA con riesgo incierto de sesgos de selección, de notificación y de cegamiento de los participantes, el personal y los evaluadores. Al mismo tiempo, la certeza proveniente de estudios no aleatorizados para este desenlace fue considerada muy baja. Por otra parte, la evidencia relativa a la seguridad de las intervenciones fue muy baja, ya que los resultados fueron únicamente aportados por estudios observacionales con un riesgo grave de sesgo en al menos dos de sus dominios (sesgo de confusión y sesgo de medición de los resultados).

Analizando la validez externa de este estudio, si bien en Argentina desde enero de 2021 entró en vigencia la Ley Nacional 27.610 , que tiene como objeto regular el acceso a la interrupción voluntaria del embarazo (IVE) y la atención postaborto ${ }^{3}$, encontramos varios aspectos a destacar. Por un lado, aún no se encuentra aprobado por la agencia reguladora de medicamentos (ANMAT) el uso de la mifepristona en nuestro país, motivo por el cual los esquemas para la interrupción del embarazo locales utilizan misoprostol solo. Por otro lado, la revisión resumida aporta evidencia únicamente para personas gestantes mayores de edad y con edades gestacionales menores a 9 semanas. Dado que en Argentina los datos estadísticos muestran una alta tasa de abortos en adolescentes ${ }^{4}$ y que la ley avala la IVE hasta las 14 semanas de gestación, sería conveniente contar con estudios que evalúen población con estas características para ejercer la práctica autoadministrada con mayor seguridad. Sin embargo, tras una búsqueda bibliográfica exhaustiva no se recuperaron estudios que incluyan personas gestantes con edades gestacionales mayores a 9 semanas o que evalúen el misoprostol como droga única, ni estudios realizados en América Latina.

\section{Conclusiones del comentador}

Si bien el aborto medicamentoso autoadministrado mostró diferencias en su eficacia respecto al supervisado por profesionales, aún resta demostrar su seguridad. Al mismo tiempo, queda pendiente evaluar la eficacia, seguridad y aceptabilidad en personas gestantes más allá del primer trimestre, en menores de 18 años, en ausencia completa de profesionales de la salud y con estrategias farmacológicas que involucren solamente el misoprostol.

Angeletti E. Eficacia y seguridad del aborto medicamentoso auto-administrado versus administrado por profesionales de la salud . Evid Actual Pract Ambul. 2021;24(2):e002127. Comentado de: Gambir K, et al. Self-administered versus provider-administered medical abortion. Cochrane Database Syst Rev . 2020 Mar 9;3(3):CD013181. PMID: 32150279 


\section{Referencias}

1. Gambir K, Kim C, Necastro KA, et al. Self-administered versus provider-administered medical abortion. Cochrane Database Syst Rev. 2020;3(3):CD013181. Available from: 10.1002/14651858.CD013181.pub2.

2. Organización Mundial De La Salud, Departamento de Salud Reproductiva e Investigaciones Conexas. Aborto sin riesgos: guía técnica y de políticas para sistemas de salud; 2012. Available from: https://www.who.int/reproductivehealth/publications/unsafe_abortion/9789241548434/es/.

3. Discacciati V, Epstein D, Musarella N, et al. En 2020, el aborto en Argentina salió de la clandestinidad. Evid Actual Pract Ambul. 2021;24(1):21152115. Available from: 10.51987/EVIDENCIA.V24I1.6910;http://www.evidencia.org.ar/index.php/Evidencia/article/view/6910.

4. Bott S, Guedes A, Goodwin M, Organización Panamericana de la Salud. Violencia contra la mujer en América Latina y el Caribe: análisis comparativo de datos poblacionales de 12 países; 2014. Available from: https://oig.cepal.org/es/documentos/violencia-mujeres-america-latina-caribe-analisiscomparativo-datos-poblacionales-12. 